\title{
A Combination of Traditional and Polarimetric Features for Oil Spill Detection Using TerraSAR-X
}

\author{
Suman Singha, Member, IEEE, Rudolf Ressel, Member, IEEE, Domenico Velotto, Member, IEEE, \\ and Susanne Lehner, Member, IEEE
}

\begin{abstract}
Synthetic aperture radar (SAR) images are operationally used for the detection of oil spills in the marine environment, as they are independent of sun light and weather-induced phenomena. Exploitation of radar polarimetric features for operational oil spill detection is relatively new and until recently those properties have not been extensively exploited. This paper describes the development of a oil spill detection processing chain using coherent dual-polarimetric (copolarized channels, i.e., HHVV) TerraSAR-X images. The proposed methodology focuses on offshore platform monitoring and introduces for the first time a combination of traditional and polarimetric features for objectbased oil spill detection and look-alike discrimination. A total number of 35 feature parameters were extracted from 225 oil spills and 26 look-alikes and divided into training and validation dataset. Mutual information content among extracted features have been assessed and feature parameters are ranked according to their ability to discriminate between oil spill and look-alike. Extracted features are used for training and validation of a support vector machine-based classifier. Performance estimation was carried out for the proposed methodology on a large dataset with overall classification accuracy of $90 \%$ oil spills and $80 \%$ for look-alikes. Polarimetric features such as geometric intensity, copolarization power ratio, span proved to be more discriminative than other polarimetric and traditional features.
\end{abstract}

Index Terms-Feature extraction, feature ranking, near real time (NRT) services, polarimetric oil spill detection, support vector machine.

\section{INTRODUCTION AND POLARIMETRIC OIL SPILL DETECTION}

$\mathbf{O}$ PERATIONAL monitoring activities (e.g., European Maritime Safety Agency's CleanSeaNet service) show regular occurrence of accidental and deliberate oil spills [1]. In the last decade, a number of semiautomatic and automatic techniques have been proposed in order to differentiate oil spill and look-alike dark spots based on single pol (HH or VV, multilooked ground range projected) synthetic aperture radar (SAR) images. Some of the proposed techniques are also capable of providing results in near real time (NRT) [1]. It is worth to note that the NRT environment for SAR oceanographic applications means delivery of the final product within $30 \mathrm{~min}$ after the image acquisition time. However, such techniques suffer from a high

Manuscript received May 04, 2015; revised August 31, 2015, November 27, 2015, February 16, 2016, and April 05, 2016; accepted April 26, 2016. (Corresponding author: Suman Singha.)

The authors are with the Maritime Safety and Security Lab, Remote Sensing Technology Institute (IMF), German Aerospace Center (DLR), 28199 Bremen, Germany (e-mail: Suman.Singha@dlr.de; Rudolf.Ressel@dlr.de; Domenico. Velotto@dlr.de; Susanne.Lehner@dlr.de).

Color versions of one or more of the figures in this paper are available online at http://ieeexplore.ieee.org.

Digital Object Identifier 10.1109/JSTARS.2016.2559946 misclassification rate which is undesirable for operational services. In addition to that, small operational spillages (production water) from offshore platforms are often ignored as its signature appears insignificant on traditional "ScanSAR" wide swath images. In order to mitigate this situation, a major focus of research in this area is the development of automated algorithms based on polarimetric images to distinguish oil spills from "look-alikes." Traditional oil spill detection techniques use backscatter, geometry, shape, or context-based feature parameters in order to classify dark-spots on an object basis. A comprehensive review of traditional oil spill detection methodologies and evaluation of traditional features can be found in [1] and [2], respectively. On the other hand, numbers of polarimetric features have been proposed for the same purpose for pixel-based classification [3]-[7] and discussed briefly in Section II.

Space-borne SAR sensors with polarimetric (dual (HH-VV) and/or quad) capabilities are now operationally available in L (ALOS-2 PALSAR), C (RADARSAT-2, RISAT), and X (TerraSAR-X, COSMO SkyMed) band and proven to be suitable for oil spill detection. Methodologies for oil spill detection systems based on polarimetry are evolving rapidly and several previous studies investigated the capability of dual copolarized SAR measurements for oil spill detection [5]-[9]. Although previous studies based on single polarimetric images manage to produce reasonable classification results using traditional features (feature based on geometry, backscatter, contextual, etc.), it may suffer from a considerable false positive rate [1], hence warrant an investigation into the polarimetric domain. Recent studies show unique benefits of polarimetric SAR data to both observe oil slicks and discriminate between oil spill and lookalike spots, which is a major challenge for traditional oil spill detection systems based only on single polarized SAR images [3], [4], [9]-[12]. Nonetheless, an integration of traditional and polarimetric-based features is yet to be established [13]. This study focuses on developing an object-based classifier which uses polarimetric features along with traditional features to distinguish oil spills from "look-alikes" using a dataset acquired primarily over offshore platforms. In addition to that, this study also looks into the performance of each feature for its ability to discriminate between two classes.

The paper is organized as follows. Section II gives an introduction about the polarimetric features and their mathematical definitions. Section III provides the description of the dataset used in this study. Section IV provides the summary of the proposed methodology and the processing chain along with a comprehensive scheme to analyze the discriminative power of polarimetric and traditional features. Experimental results and 
discussions about the performance of the classifier and feature analysis are presented in Section V, while summary and conclusion are drawn in Section VI.

\section{Polarimetric OIL SPILl Detection}

Polarimetric features are directly related to the physical properties of the scattering surface. As mentioned in the previous section, several researcher have demonstrated the benefits of multipolarization measurements for oil spill detection over the last decade. The polarimetric scattering characteristics of the oil-covered sea surface depends on several contributing factors. According to their importance, a list of contributing factors are given below.

1) Substance type (e.g., mixture of organic compounds, volume, age of the spilled oil).

2) SAR instrument specific parameters (e.g., polarization, incidence angle, noise floor, imaging mechanism).

3) Geophysical parameters (e.g., local wind vector, presence of capillary waves, surface currents).

Moreover, even the molecular structure and emulsification processes of the oil can affect the dielectric properties of oilcovered waters and their scattering properties.

In case of a fully polarimetric acquisition, the scattering matrix is given by

$$
S=\left[\begin{array}{ll}
S_{\mathrm{HH}} & S_{\mathrm{HV}} \\
S_{\mathrm{VH}} & S_{\mathrm{VV}}
\end{array}\right]
$$

where $S_{\mathrm{XX}}=\left|S_{\mathrm{XX}}\right| \exp \left(j \varphi_{\mathrm{XX}}\right)$, with $\left|S_{\mathrm{XX}}\right|$ representing the amplitude and $\varphi_{\mathrm{XX}}$ representing the phase of the complex scattering coefficient. Although TerraSAR-X and TanDEM-X are capable of acquiring fully polarimetric data (experimental mode), only dual polarimetric data are available operationally. In case of dual polarimetric TerraSAR-X acquisitions all possible combinations are available $\left(S_{\mathrm{HH}}-S_{\mathrm{HV}} ; S_{\mathrm{VV}}-S_{\mathrm{VH}}\right.$, and $\left.S_{\mathrm{HH}}-S_{\mathrm{VV}}\right)$. In this study, only $S_{\mathrm{HH}}$ and $S_{\mathrm{VV}}$ scattering coefficients are used in order to extract relevant polarimetric features. Therefore in this case, utilizing only copol channels, the scattering vector is given by

$$
k=\frac{1}{\sqrt{2}}\left[S_{\mathrm{HH}}+S_{\mathrm{VV}}, S_{\mathrm{HH}}-S_{\mathrm{VV}}\right]
$$

and copolarized phase difference (CPD) is given by

$$
\mathrm{CPD}=\varphi_{\mathrm{HH}}-\varphi_{\mathrm{VV}} .
$$

The concept for using polarimetric SAR (PolSAR) data for oil slick observation was initially assessed in [14] and later on in [3] for SIR-C/X-SAR data followed by a demonstration on fully polarimetric ALOS PALSAR data in [5]. The principal concept was then adapted for X-band by [9] using TerraSAR-X dualpol coherent single look complex (SLC) data. The polarimetric electromagnetic model, which is the basis of this proposed methodology, predicts that the sea surface is governed by Bragg scattering (or tilted-Bragg scattering), has a high interchannel (co-pol) correlation and in case of non-Bragg scatter (e.g., oilcovered sea surface) the correlation is considerably lower [9] [7]. In [9], the authors demonstrated that the standard deviation of CPD for a polluted area is significantly higher compared to the pollution-free background due to significant deviation from the Bragg scattering mechanism and on the other hand lower coherence for polluted area due to the same reason. In [3], [5], [7], [15], the model has been demonstrated to be valid even at different frequency bands (L-, C-, X-band) making it attractive for further exploitation. CPD has a probability distribution (pdf) that depends on two factors, the number of looks $\ell(\ell=1$ in case of TerraSAR-X Dual-Pol StripMAP data) and the complex correlation coefficient between $\mathrm{HH}$ and $\mathrm{VV}\left(\rho_{\mathrm{CO}}\right.$, given in $4)$, where ${ }^{*}$ denotes complex conjugate and $\langle\cdot\rangle$ denotes spatial averaging

$$
\rho_{\mathrm{CO}}=\frac{\left|\left\langle S_{\mathrm{HH}} S_{\mathrm{VV}}^{*}\right\rangle\right|}{\sqrt{\left\langle S_{\mathrm{HH}} S_{\mathrm{HH}}^{*}\right\rangle\left\langle S_{\mathrm{VV}} S_{\mathrm{VV}}^{*}\right\rangle}}
$$

where

$$
\left\langle S_{\mathrm{HH}} S_{\mathrm{VV}}^{*}\right\rangle=\left\langle\left|S_{\mathrm{HH}}\right|\left|S_{\mathrm{VV}}\right| e^{i\left(\varphi_{\mathrm{HH}}-\varphi_{\mathrm{VV}}\right)}\right\rangle .
$$

When $\mathrm{HH}$ and VV are uncorrelated (in case of oil covered surface), the pdf becomes uniformly distributed between $-180^{\circ}$ and $180^{\circ}$. The probability distribution of the CPD in case of oil-covered sea surface and oil-free sea surface extracted from TerraSAR-X data can be found in [9].

The coherency matrix for the dual polarization case, represented by $T^{(d)}$ is given in (6). The superscript ${ }^{(d)}$ is used throughout this manuscript to distinguish the dual-polarimetric adaptation, i.e., $T^{(d)}$

$$
\begin{aligned}
& T^{(d)}=\frac{1}{\sqrt{2}} \\
& {\left[\begin{array}{cc}
\left\langle\left|S_{\mathrm{HH}}+S_{\mathrm{VV}}\right|^{2}\right\rangle & \left\langle\left(S_{\mathrm{HH}}+S_{\mathrm{VV}}\right)\left(S_{\mathrm{HH}}-S_{\mathrm{VV}}\right)^{*}\right\rangle \\
\left\langle\left(S_{\mathrm{HH}}-S_{\mathrm{VV}}\right)\left(S_{\mathrm{HH}}+S_{\mathrm{VV}}\right)^{*}\right\rangle & \left\langle\left|S_{\mathrm{HH}}-S_{\mathrm{VV}}\right|^{2}\right\rangle
\end{array}\right] .}
\end{aligned}
$$

In [3], the well-known Eigenvalue decomposition is proposed for an oil spill detection algorithm. Eigenvalue-based polarmetric features, i.e., entropy $(H)$, anisotropy $(A)$, and mean scattering angle $(\alpha)$ [16]-[18] are used in combination with a constant false alarm rate filter for oil spill classification on a single SIR-C/X-SAR L-band dataset. In a polarimetric approach, one generally first computes the (locally averaged by $9 \times 9$ sliding window) covariance matrix of the scattering vector in order to compute the eigenvalues. The eigenvalues $\lambda_{1}$ and $\lambda_{2}$ of $T^{(d)}$ are used to compute $p_{j}=\lambda_{j} /\left(\lambda_{1}+\lambda_{2}\right)$. These are the input for determining entropy

$$
H^{(d)}=-\left(p_{1} \log \left(p_{1}\right)+p_{2} \log \left(p_{2}\right)\right)
$$

and anisotropy

$$
A^{(d)}=\frac{\left(p_{1}-p_{2}\right)}{\left(p_{1}+p_{2}\right)} .
$$

The eigenvectors $v_{1}=\left(v_{1}^{(1)}, v_{1}^{(2)}\right)$ and $v_{2}=\left(v_{2}^{(1)}, v_{2}^{(2)}\right)$ of the coherency matrix (6) provide

$$
\alpha_{i}=\arccos \left(v_{i}^{(1)}\right), i=1,2 .
$$


In this study, we used the $\alpha$ angle for the dual-polarimetric case which is defined as

$$
\alpha^{(d)}=\alpha_{1} p_{1}+\alpha_{2} p_{2} .
$$

The coherency matrix $\left(T^{(d)}\right.$, in (6)) is used as basis for target decompositions for extracting some of the features used in this study. It is worth mentioning that the coherency matrix $\left(T^{(d)}\right)$ for dual-pol TerraSAR-X data has two eigenvectors $\left(\lambda_{1}\right.$ and $\left.\lambda_{2}\right)$, and the eigenvector-based parameter used for this study is different from the traditional definition due to the unavailability of the cross-polarized channels which has a negligible effect in this scenario [12]. While the classical $H-A-\alpha$ parameters (cf. [19]) in the fully polarimetric case also permit the physical interpretation (e.g., about predominant scattering mechanisms and range values between 0 and 1), it is important to note that the two-dimensional coherency matrix does not capture the full physical phenomena of the scatterer, but only part of it. This entails a possible loss of the physical meaning of the subsequent definition of $H-A-\alpha$ parameters. In case of oil-covered sea surface, $H$ tends to zero, therefore it is a dominant scattering mechanism scenario. On the other hand, pollution-free sea surface is dominated by relatively high entropy, representing random scattering mechanisms which is also true for the dualpolarimetric adaptation [6], [20].

Entropy has also been found useful for oil spill detection on ALOS PALSAR polarimetric data in [21]. In [22], the authors also used a combination of Cloude-Pottier-based decomposition features $(H-A-\alpha)$ along with the copol correlation coefficient $\left(\rho_{\mathrm{CO}}\right)$ in order to form a feature combination to enhance oil spill detection capability on UAVSAR L-band fully polarimetric data. In addition to that, polarimetric Span is also investigated as a separate feature in that study. Polarimetric Span can be derived from the summation of eigenvalues, therefore, in dualpolarimetric case it is given by

$$
\operatorname{Span}^{(d)}=\lambda_{1}+\lambda_{2}
$$

A recent study [6] evaluated eight well-established and modified polarimetric features on two C-band RADARSAT-2 fine quad pol datasets. In that study, geometric intensity, which is expressed as

$$
\mu^{(d)}=\operatorname{det}\left(T^{(d)}\right)^{1 / 2}
$$

is used as a polarimetric feature based on the $T^{(d)}$ coherency matrix as a replacement for polarmetric $\operatorname{Span}^{(d)}$. That study claimed based on empirical results that $\mu^{(d)}$ is superior to $\operatorname{Span}^{(d)}$ as $\mu^{(d)}$ utilizes all elements of $T^{(d)}$, i.e., information on the cross products in addition to the main diagonal entries used for the $\operatorname{Span}^{(d)}$. As expected from (12), dark spots have relatively low value of $\mu^{(d)}$ compared to the background. In addition to the $T^{(d)}$ matrixbased features, CPD, $\rho_{\mathrm{CO}}$ and two additional lexicographicbased features, the copolarization power ratio $\left(\gamma_{\mathrm{CO}}\right)$ and real part of the copolarization cross product $(R \mathrm{co})$, are evaluated in [6]. The copolarization power ratio, is given by the ratio between amplitudes of the complex scattering coefficients in the $\mathrm{HH}$ and
TABLE I

Traditional [23] AND POLARIMETRIC FEATURES USED FOR THIS STUdY

\begin{tabular}{ll}
\hline \hline Traditional Features & Polarimetric features \\
\hline Area $\left(\mathrm{km}^{2}\right)$ & Stddev Co-Pol Phase Difference $\sigma_{\varphi, \mathrm{CO}}[9],[5]$ \\
Perimeter $(\mathrm{km})$ & Coherency Coefficient $\rho_{\mathrm{C} O}[9]$ \\
Complexity (dimensionless scalar) & Real Part Co-Pol Cross Product $R$ co [5] \\
Spreading (percentage) & Polarimetric Span ${ }^{(d)}[20]$ \\
Sddev Object (StdDev_Obj) & Copol Power Ratio $\gamma_{\mathrm{CO}}[6]$ \\
Max Contrast (Max_Contr) & Geometric Intensity $\mu^{(d)}[6]$ \\
Mean Contrast (Mean_Contr) & Entropy $H^{(d)}[6],[20]$ \\
& Anisotropy $A^{(d)}[6],[20]$ \\
& Alpha angle $\alpha^{(d)}[6],[20]$ \\
\hline \hline
\end{tabular}

VV polarization channels

$$
\gamma_{\mathrm{CO}}=\frac{\left\langle\left|S_{\mathrm{HH}}\right|^{2}\right\rangle}{\left\langle\left|S_{\mathrm{VV}}\right|^{2}\right\rangle}
$$

The copolarization ratio is generally higher over darkspots (specially over oil) compared to the background and that is partially due to a lower dielectric constant of oil compared to sea ([6] and [11]).

The real part of the copolarization cross product $(R \mathrm{co})$ is given by

$$
R \mathrm{co}=\left|\Re\left(\left\langle S_{\mathrm{HH}} S_{\mathrm{VV}}^{*}\right\rangle\right)\right|
$$

where $\Re$ stands for the real part. $R$ co is initially proposed for oil spill and look-alike discrimination in [15] (also in [21]) as part of a filter, where oil spills are distinguished from clean sea and biogenic look-alikes with the help of the ratio between $R$ co and $\left\langle\left|S_{\mathrm{HV}}\right|^{2}\right\rangle$. In [15], authors described the physical rationale behind this fact which is, sea surface covered with biogenic look-alikes still dominated by Bragg scattering, hence indistinguishable from clear background. In case of an oil-covered area, the value of $R$ co is expected to be nearly zero and in case of a clear background $R$ co is expected to have a value greater than zero [15].

The proposed methodology uses a combination of wellknown traditional features used for operational services [23] and recently established polarimetric features to characterize dark spots. All of the features used in this study are given in Table I. Details of the traditional features used in this study can be found in [23].

\section{DATASET}

The majority of the images used to develop and validate the proposed algorithm were acquired over Bombay High near the west coast of India, hosting a cluster of offshore oil platforms. Unlike European waters, there are no observations derived from regular airborne surveillance, so the only possible way to monitor this kind of area is the use satellite SAR imagery. Bombay High is an offshore oilfield, 160 $\mathrm{km}$ off the coast of Mumbai, India, with about $75 \mathrm{~m}$ waterdepth and in production since 1974. All of the images acquired are TerraSAR-X SLC dual pol (HH and VV) StripMAP products with slant range resolution of $1.17 \mathrm{~m}$ which is ideal for monitoring offshore platform clusters like Bombay 


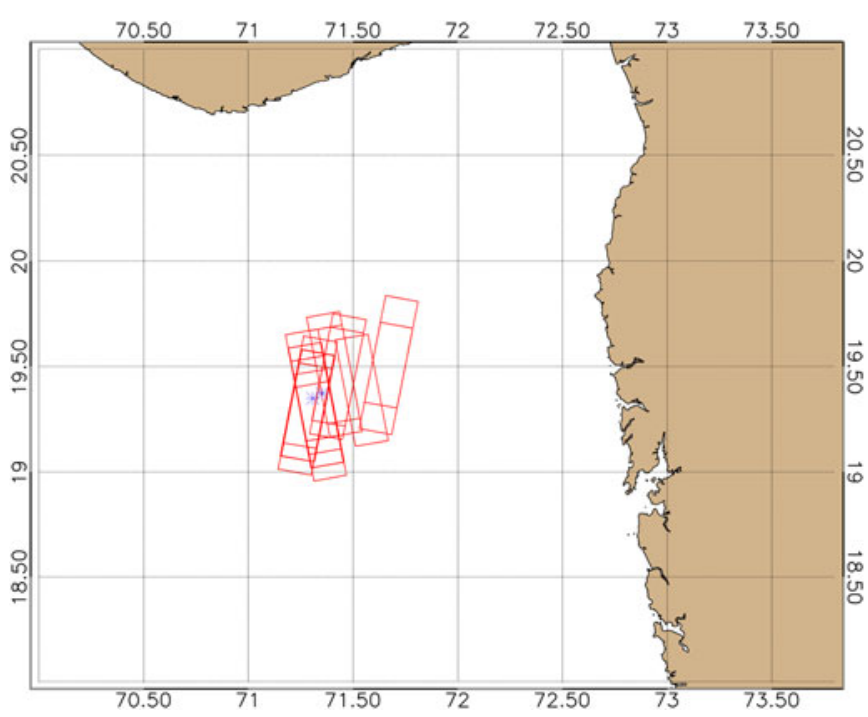

Fig. 1. Footprints of TerraSAR-X dual polarimetric (HH-VV, consecutive ascending and descending pass) images acquired over Bombay High offshore platform cluster indicated with red rectangles (approximate location of platfrom clusters are shown in blue marks).

High. Fig. 1 shows the location of the study area, platform locations and an overview of the acquired TS-X images. Starting in February 2014 and continuing until the end of 2014, a total number of 48 images were acquired over the platform cluster with same day ascending and descending configuration. The dataset includes different beams of TerraSAR-X StripMAP mode (e.g., StripFar_006, StripNear_009, etc.) incorporating a broad range of incidence angles, from $20^{\circ}$ to $35^{\circ}$. This incidence angle range was chosen due to its low instrument noise floor (NESZ range $[-19 \mathrm{~dB} ;-26 \mathrm{~dB}]$ ) which is a critical parameter in respect to the polarimetric system performance [9]. Wind speed estimation was carried out on each image (on VV polarized channel) using the XMOD2 algorithm [24]. A wind speed range of 3-14 m/s was chosen to obtain the final dataset used in this study and few images were discarded from the initial dataset due to low wind condition. Thereafter, the dataset went through manual interpretation technique to identify oil spill and lookalike spots in order to prepare training and validation dataset. The dataset is strongly heterogeneous, containing 226 oil spill spots, providing fresh and old platform sourced spills and incorporating different wind conditions. Moreover, the dataset contains a comprehensive set of look-alikes (26), such as ship wakes, low wind areas, and rain cells. A small number of lookalikes present in the data are not from the study area. Fig. 2 shows an examples of several platform sourced oil spills on a TS-X StripMAP dual pol (HH-VV, descending orbit) acquired on April 20, 2014 at 01:15 UTC over the study area. Fig. 2 also shows us the presence of several offshore installation scattered throughout the upper portion of the image.

The overall dataset which contains a total number of 252 feature vectors representing both oil spill and "look-alike" classes are then divided into two datasets, training and validation dataset. The training dataset contains 142 feature vectors representing 126 oil spill and 16 look-alike spots, on the

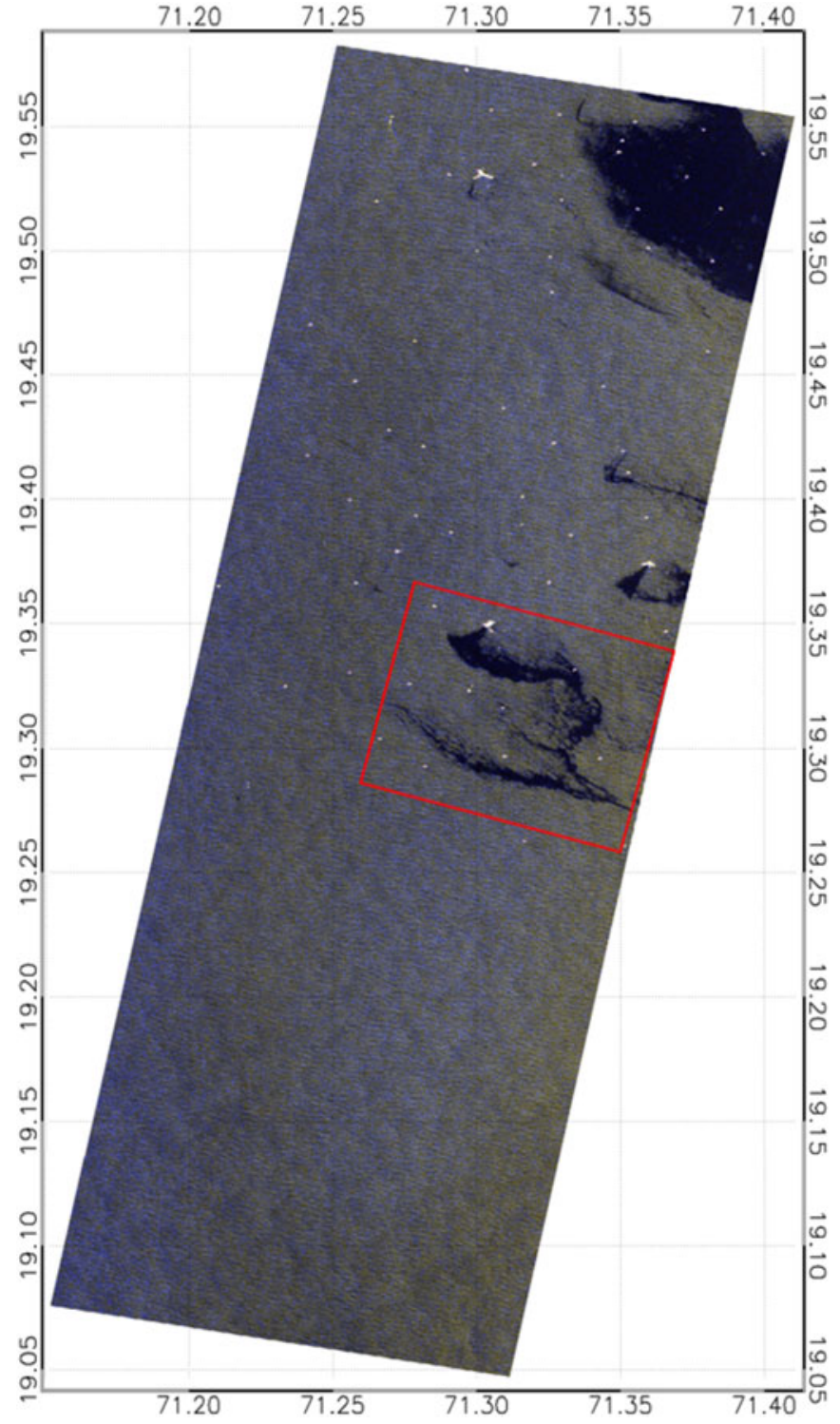

Fig. 2. TerraSAR-X dual polarimetric (red-HH, green-VV, blue-HH-VV, mid incidence angle $\theta=29.37^{\circ}$ ) StripMAP (stripFar_006) image over Bombay High offshore platform cluster. One example of ROI is indicated with red rectangle.

other hand the validation dataset consists of 110 feature vectors representing 100 oil spills and 10 look-alike spots. It is important to note that the training and validation datasets are mutually exclusive. Initially the training and the validation dataset went through manual/visual interpretation of SAR images in order to prepare the reference target.

\section{Methodology}

A complete overview of the proposed methodology is shown in Fig. 3 where the left-hand side of the figure shows the flowchart of the proposed methodology and the right-hand side of the figure shows major steps involved in the proposed methodology, i.e., segmentation of a dark spot, extracted features and final output. First step of the proposed methodology involves standard preprocessing (including product metadata retrieval, 


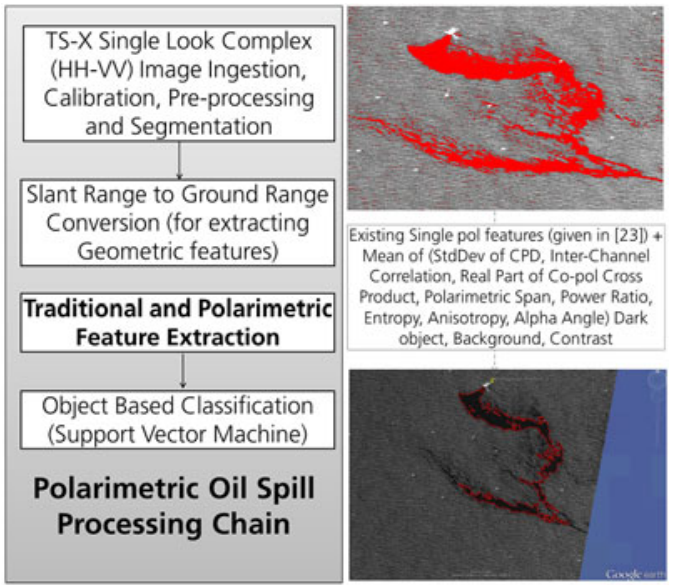

Fig. 3. Methodology for near real time oil spill detection processing chain based on dual polarimetric TerraSAR-X images. The upper right image represents the segmentation precess and the lower right image shows the final result where the detected oil spill is indicated with red polygon.

geo-referencing, land-masking, and speckle removal) and calibration. After that, a segmentation process is carried out using adaptive thresholding technique, based on VV polarized channel $\left(\sigma_{0 \mathrm{Vv}}\right)$ in order to extract the dark object present within the "Region of Interest." Then, from each segmented dark spot, a set of traditional features (given in [23], see Table I) and a set of polarimetric features (see Table I and Fig. 4) are extracted. A sliding window of size $9 \times 9$ is used for extracting polarimetric features, as this window size was found to be a good trade-off between speckle reduction and preservation of resolution. It is important to note here that the segmentation process is one of the essential parts of the methodology and affects the overall performance of the methodology, therefore here we used an already established methodology reported in [23]. Mean of dark spot, mean of background and mean contrast between dark spot and background are taken into account for each polarimetric feature. In order to further elaborate mean contrast, for a given polarimetric feature $\mathrm{PF}$, the contrast between background and dark-spot in SAR imagery, i.e., $\zeta_{\mathrm{PF}}$, can be defined as the ratio of the mean value of a dark-spot extracted from "Region of Interest" to the mean value of the background sea surface without the dark spot (within the "Region of Interest"), therefore

$$
\zeta_{\mathrm{PF}}=\frac{\left\langle\mathrm{PF}_{\text {darkspot }}\right\rangle}{\left\langle\mathrm{PF}_{\text {background }}\right\rangle}
$$

Furthermore, we assumed that features related to background do not carry any class discriminative power by themselves and most of the time, they are unrelated to dark features. Therefore, features related to background are only used for generating contrast-related features (see (15)), but are not included in the final feature set (Stddev background from traditional feature set was also excluded). We only considered a total number of 25 features which include 18 polarimetric and 7 traditional features for classification stage. Fig. 4 shows us different polarimetric features (described in Section II) extracted from a region of interest indicated with a red rectangle in Fig. 2. These imagettes give a visual impression of the polarimetric behavior (i.e., the different features) in one particular instance (oil spill), which differ for other dark spot detections. On these differences in the polarimteric features of dark spots we will base our classification.

These extracted feature parameters are then used for training and calibration of the support vector machine (SVM), which is designed to discriminate between oil spill and look-alike spots. Although two-class classification is very common in case of oil spill-"look-alike" discrimination, a study by [25] proposes one-class classification (outliers detection problem) for this scenario. There authors pointed out that "look-alikes" itself do not constitute a single class, as they are created by different physical phenomena. In our case, we adapt a two-class classification approach as our training and validation dataset contain a comprehensive and representative array of "look-alike" samples (occurring due to the presence of different physical phenomena). Moreover two-class classifiers managed to produce excellent results in operational scenarios [1]. In case of the two-class approach, the SVM embeds (nonlinearly) the original feature vector into a high-dimensional feature space in order to find an optimal separating criterion between classes. The SVM classifier is especially useful for ill-posed problems [26],[27]. A common ill-posed problem arises when dealing with datasets with a low ratio between a number of samples and the feature space dimension. Since the extraction of our array of features constitutes a mapping from a space of four real dimensions (two complex dimensions) to 25 real dimensions, the mapped data clusters can be assumed to be low dimensional as well even in our high-dimensional feature space. For this reason, we do not expect the Hughes phenomenon to strongly affect our algorithm. The SVM classification for oil spill and look-alike was implemented and evaluated with satisfactory result in [27]. The SVM is initially trained with the training dataset and validated further with a mutually exclusive validation dataset described in the previous section. After a decision has been made whether a dark spot is either oil spill or look-alike, a delivery product (e.g., $\mathrm{kml}$ or shapefile format) is generated according to the user requirement. The final product usually includes a reduced resolution ground range projected image and detailed information regarding the oil spill polygon. A detailed classification result using the validation dataset obtained from the trained SVM classifier is given in Section V.

Besides the overall classification accuracy, naturally the focus also lies on finding out the contribution of particular features and/or feature combinations to the classification result. Therefore, we carried out a mutual information analysis on extracted features which include both training and testing dataset. One common approach of a comprehensive statistical investigation is to analyze redundancies and relevances in terms of mutual information(see [28], [29]). Besides the theoretical interest, such a statistical analysis into features' usefulness generally is conducted to reduce feature dimension. The dimensionality (i.e., number of features) is not the biggest obstacle in the current scenario, but rather the image size. When redundancy or lack of relevance allows to discard certain features before the classification, the computational overhead and memory consumption can 

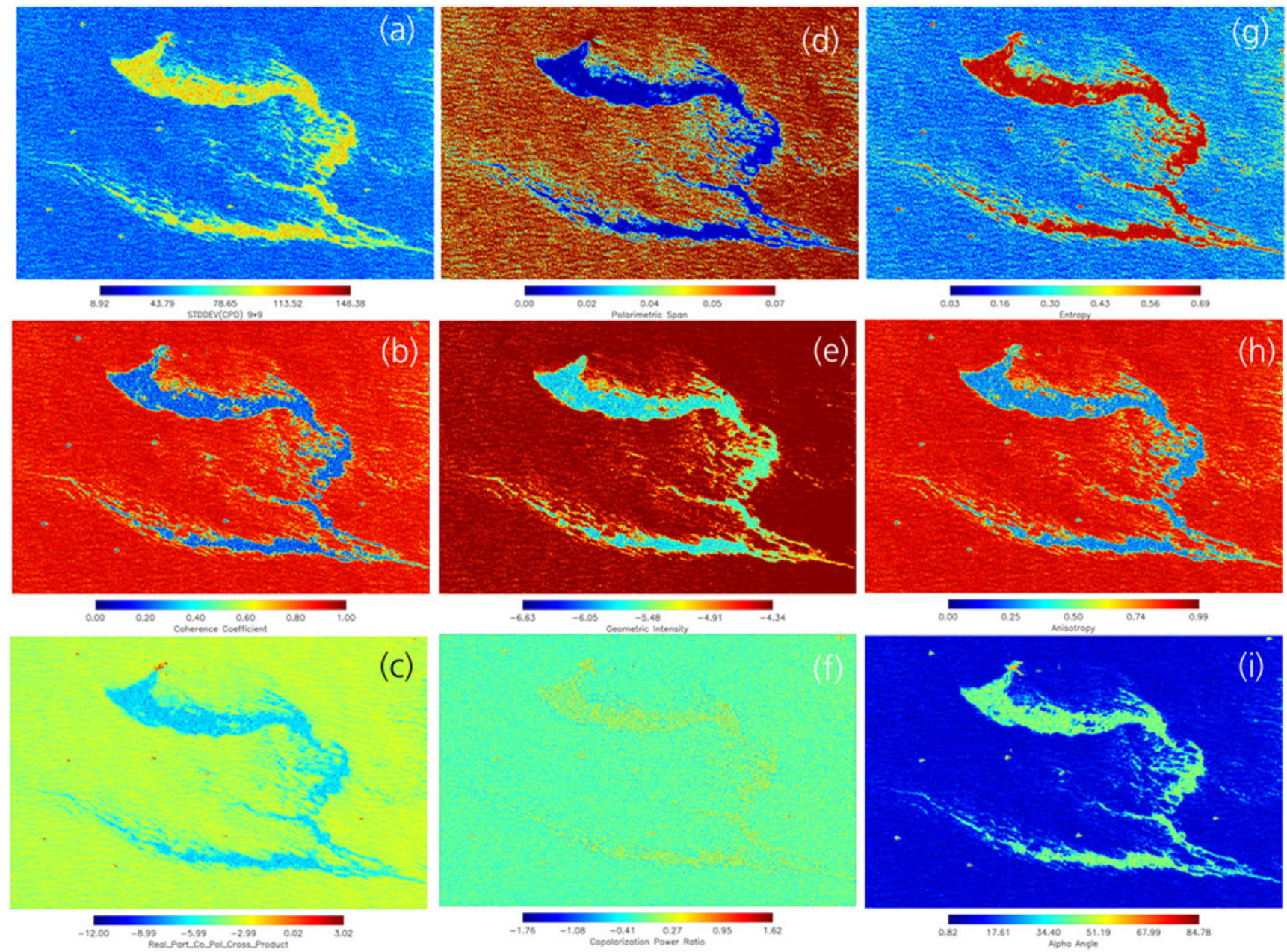

Fig. 4. Polarimetric features extracted from TerraSAR-X (HH-VV) subscene containing an oil spill spot indicated in Fig. 2. (a) Standard deviation of copolarization phase difference, $\sigma_{\varphi_{\mathrm{CO}}}$. (b) Magnitude of coherence coefficient $\rho_{\mathrm{CO}}$. (c) Real part of the copolarization cross product, $\left(\log \left(r_{\mathrm{CO}}\right)\right)$ ) (d) Polarimetric $\operatorname{Span}{ }^{(d)}$ (e) Geometric intensity, $\log \left(\mu^{(d)}\right)$. (f) Copolarization power ratio, $\log \left(\gamma_{\mathrm{CO}}\right)$. (g) Entropy, $H^{(d)}$. (h) Anisotropy, $A^{(d)}$. (i) Alpha angle $\alpha^{(d)}$. The features $\mu^{(d)}, \gamma_{\mathrm{CO}}, r_{\mathrm{CO}}$ are $\log$ transformed for visualization purpose.

be greatly reduced. Such considerations about prudent management of data quantity become ever more relevant, since modern SAR sensors produce huge datasets (TerraSAR-X SLC SM: 0.9 GB; sentinel SLC IW: 9GB) and any additional feature adds another image layer that needs to be computed and stored.

Given two random variables $\mathrm{X}, \mathrm{Y}$ (with joint and marginal densities, no point masses), the mutual information $\mathcal{I}$ of these variables is defined as

$$
\mathcal{I}(X \mid Y)=\sum_{y \in Y} \sum_{x \in X} p(x, y) \log \left(\frac{p(x, y)}{p(x) p(y)}\right)
$$

where $p(x)$ denotes the probability distribution of $X$ (likewise $p(y)$ denotes the distribution for $Y)$, and $p(x, y)$ the joint density of $X$ and $Y$. For theoretical details, see [28], [29].

Intuitively, $\mathcal{I}$ can be said to describe the portion of information that is shared by both, $X$ and $Y$, i.e., their information overlaps. Put differently, the higher $\mathcal{I}(X \mid Y)$, the more information one can infer about $X$ from existing knowledge of $Y$. In this way, one can measure in an information theoretical sense, the (nonlinear) information correlation of $X$ and $Y$. The symmetry of $\mathcal{I}$ in
$X$ and $Y$, which one would expect for the intuitive concept of "shared information," also holds for the strict mathematical definition, i.e., $\mathcal{I}(X \mid Y)=\mathcal{I}(Y \mid X)$. Given $\mathcal{I}$ is a dimensionless quantity, we use it only as a means to put different features in comparison. The absolute value of $\mathcal{I}$ we do not investigate further here.

When $X$ is chosen to be the class property and $Y$ some polarimetric feature, the mutual information $\mathcal{I}(X \mid Y)$ indicates how well one already knows about the class once information of feature $Y$ is present. Therefore, in this constellation, mutual information is capable of indicating the classification relevance of one particular feature. In case both features $Y_{1}$ and $Y_{2}$ are about equally relevant for determining class property $X$ (i.e., $\mathcal{I}\left(Y_{1} \mid X\right) \approx \mathcal{I}\left(Y_{2} \mid X\right)$ ), high mutual information $\mathcal{I}\left(Y_{1} \mid Y_{2}\right)$ then indicates redundancy.

Regarding the feature analysis we include all contrast features and the feature values obtained for the objects (i.e., xxx_Obj). The feature values for the background do not carry any class discriminative power by themselves, only in conjunction with those for the object (in order to compute xxx_contr features). After 


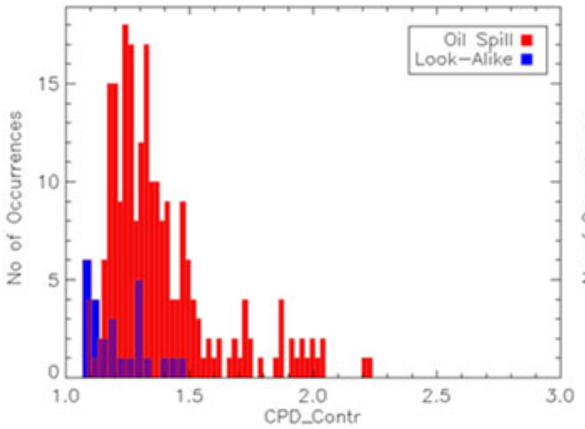

(a)

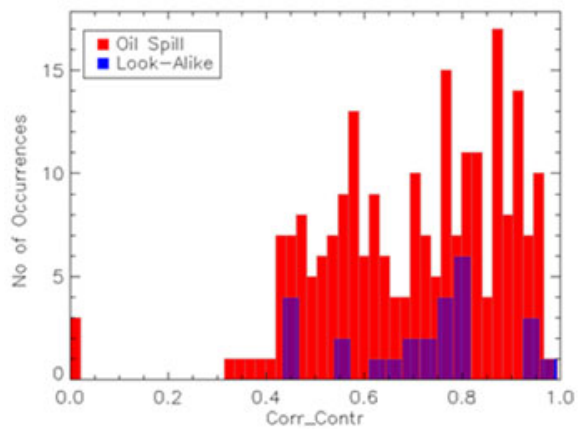

(b)

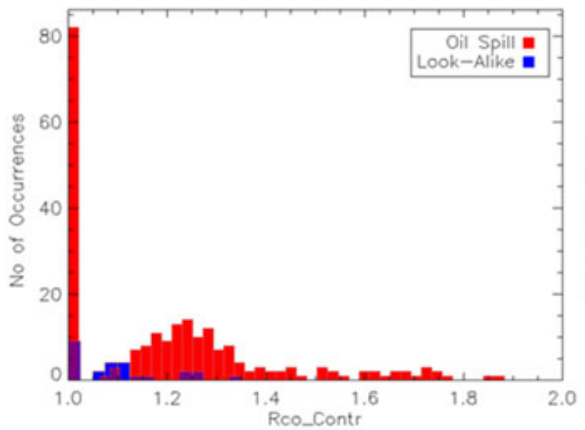

(c)

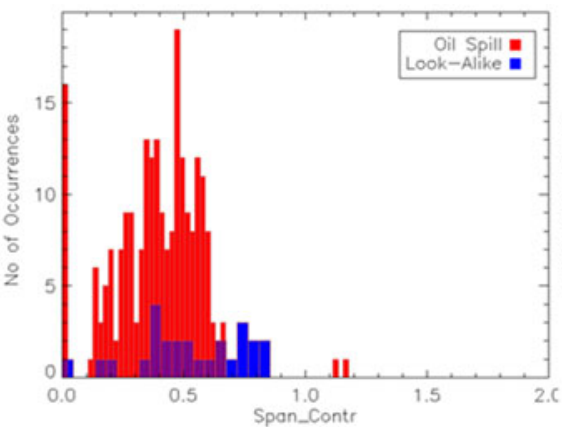

(d)

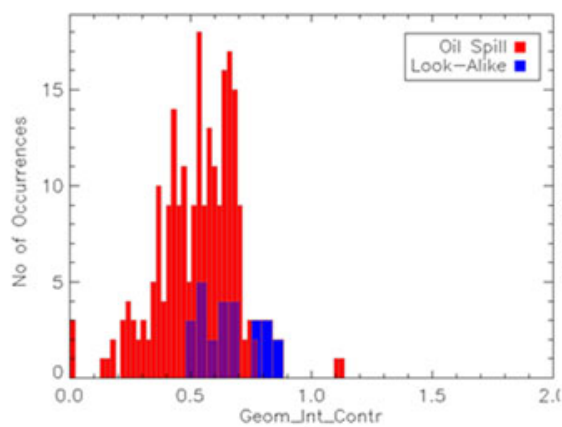

(e)

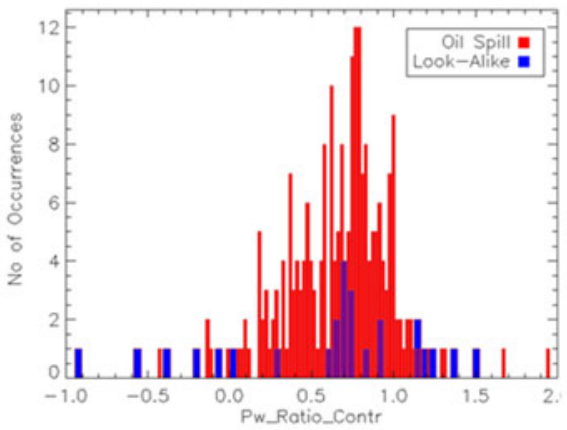

(f)

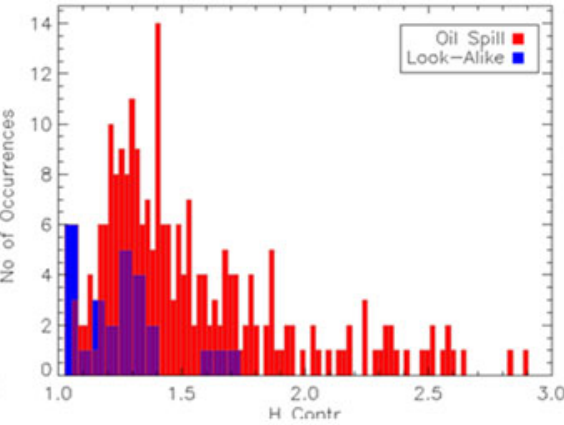

(g)

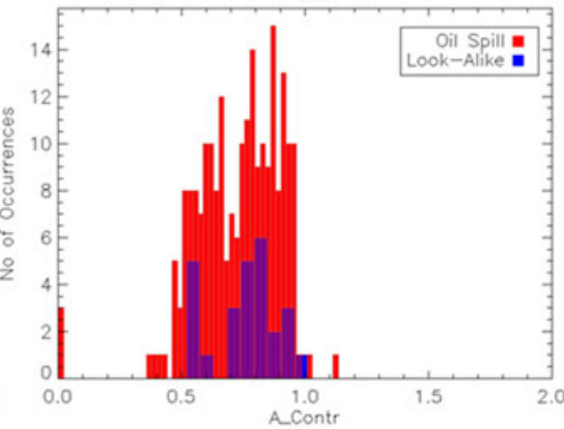

(h)

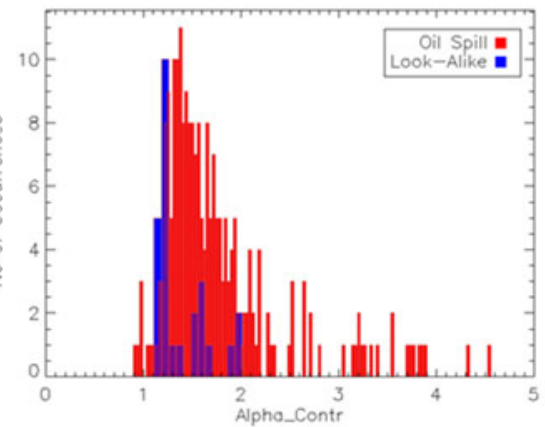

(i)

Fig. 5. Distribution of polarimetric features (mean contrast between dark-spot and background) extracted from TS-X (HH-VV) dataset, Red: oil spill, Blue: look-alike. (a) Standard deviation of co-polarization phase difference, $\sigma_{\varphi_{\mathrm{CO}}}$. (b) Magnitude of coherence coefficient $\rho_{\mathrm{CO}}$. (c) Real part of the copolarization cross product, $\log \left(r_{\mathrm{CO}}\right)$. (d) Polarimetric $\operatorname{Span}^{(d)}$ (e) Geometric intensity, $\log \left(\mu^{(d)}\right)$. (f) Copolarization power ratio, $\log \left(\gamma_{\mathrm{CO}}\right)$. (g) Entropy, $H^{(d)}$. (h) Anisotropy, $A^{(d)}$. (i) Alpha angle $\alpha^{(d)}$. Histogram bins for oil spill and look-alike were adjusted for visualization purpose.

including the object and contrast features, the background features are redundant and excluded from the final feature set (including Stddev Background from traditional feature set). Detailed results regarding mutual information analysis are presented in Section V-B.

\section{RESULTS AND DISCUSSIONS}

A methodology for the detection of oil spills from X-band dual polarimetric SAR imagery was described in the previous section. This section will provide an evaluation of classification performance of the SVM, along with a quantitative analysis on the polarimetric and traditional features combining training and validation datasets, i.e., 252 feature vectors representing 226 oil spill and 26 look-alike spots. Fig. 5 shows the distribution of each polarimetric feature $\left(\zeta_{\mathrm{PF}}\right)$ used in this study where the red bar shows distribution of oil spill and the blue bar shows distribution of look-alike spots. While Fig. 4 visualized how we characterize each dark spot by different character traits (features), the distributional synopsis over all samples and all features in Fig. 5 shows that each such feature is by itself not capable of distinguishing oil spills from look-alikes. One rather concludes from Fig. 5 that a combination of feature parameters should be used for oil spill and look-alike classification as one particular feature is unable to create enough separation between two classes: The overlap of the distributions of oil spills and look-alike is so prevalent for any of the features that one cannot possibly identify a threshold to separate the two classes. Only by considering the entire array of features one can hope to locate distinct data clusters in the high-dimensional feature space. To analyze the discriminative power, the secondary focus of this section will be an investigation of the information content of the 


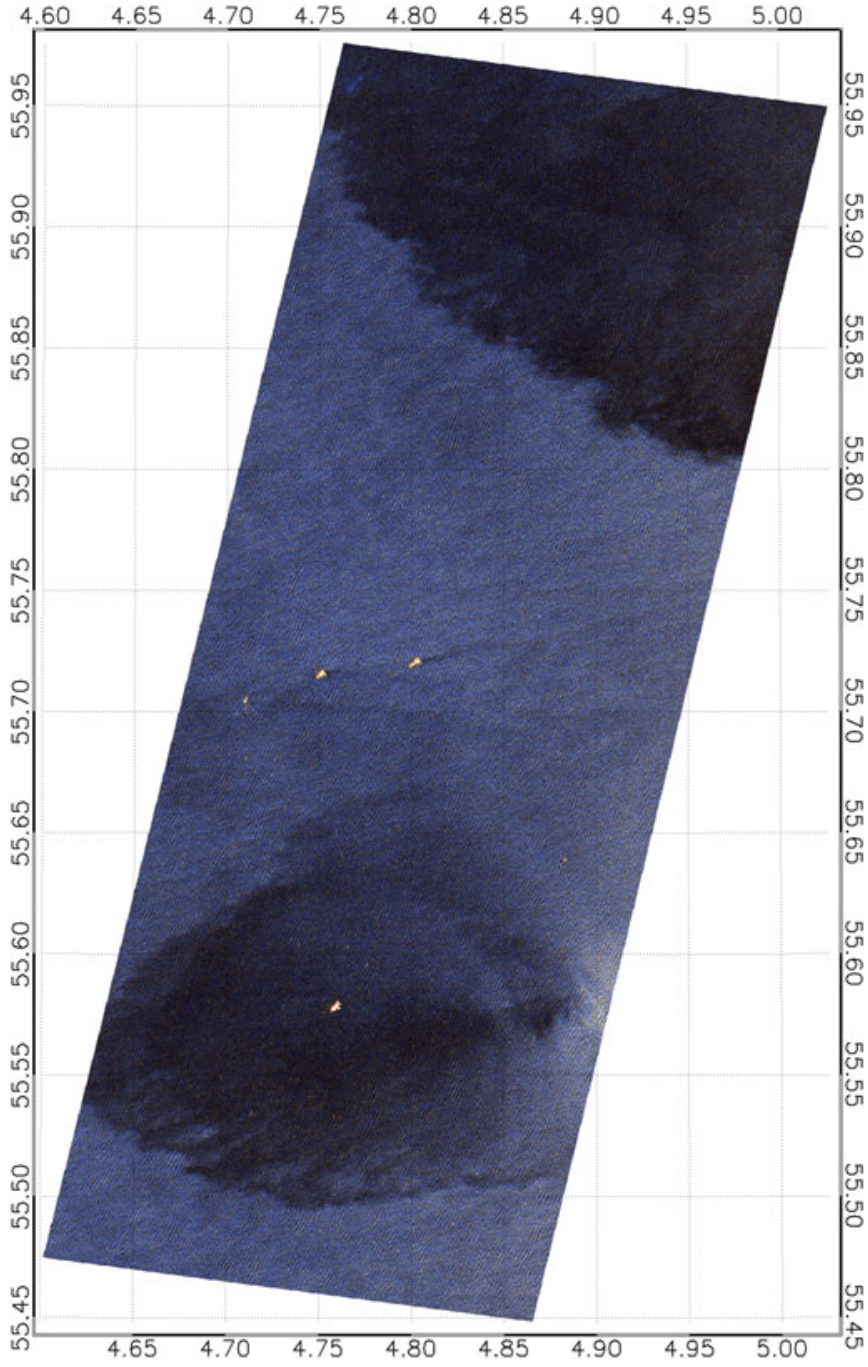

Fig. 6. TerraSAR-X Dual polarimetric (HH-VV) StripMAP image showing weather related look-alike phenomenon.

individual features and possible redundancy in terms of mutual information content among different features.

As mentioned earlier, the most crucial part of the oil spill detection process is to differentiate between oil spills and lookalikes. Fig. 6 shows an example of a weather-induced (probably due to rain cells) look-alike phenomenon along with some small look-alikes produced by wind shadow (immediate vicinity of platforms present at the center of the image) on a TerraSAR-X $(\mathrm{HH}-\mathrm{VV})$ image acquired in a relatively higher incidence angle. Two large dark spots are most likely due to rain cells (one at the bottom of the image and another at the top right portion of the image) and have higher dampening effects than ship wakes or biogenic substances. This kind of look-alike produces similar radar backscatter compared to oil spill, however, with the help of combined feature set those dark spots were classified as lookalikes. Fig. 7 shows an example of a dark spot classified as oil spill. With the help of contextual information (presence of confirmed offshore platforms), it can be concluded that this is offshore platform scoured pollution which is very frequent in this particular geographic area.

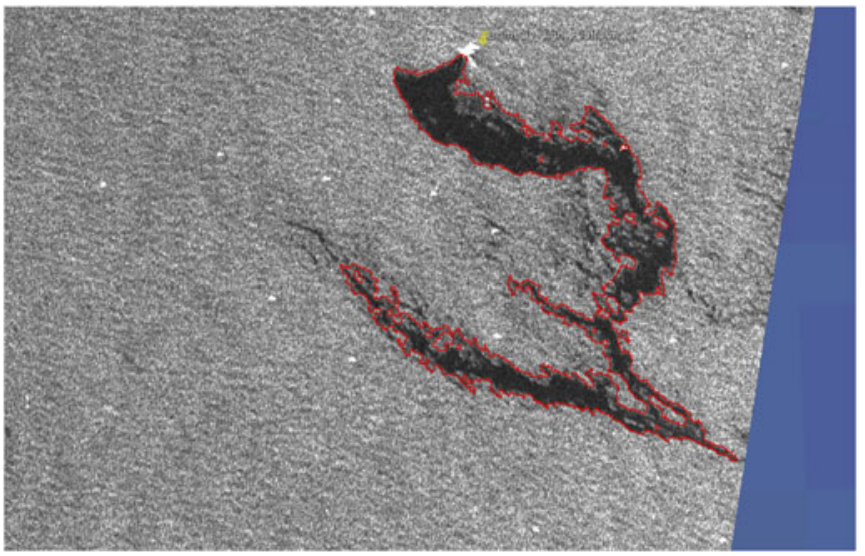

Fig. 7. Detected oil spill (represented with red polygon) on TerraSAR-X dual polarimetric images acquired over Bombay High offshore platform cluster. Presented background SAR image is VV polarized.

TABLE II

FRAMEWORK OF THE CONFUSION MATRIX USED FOR QUANTITATIVE ASSESSMENT OF THE PROPOSED ALGORITHM USING COMBINATION OF Traditional and Polarimetric Features (ALGO: Proposed ALGORITHM; REF: REFERENCE DATASET)

\begin{tabular}{lcc}
\hline \hline & Oil Spill ALGO & Look-Alike ALGO \\
\hline Oil Spill REF & $A=90,(90 \%)$ & $B=10,(10 \%)$ \\
Look-Alike REF & $C=2,(20 \%)$ & $D=8,(80 \%)$ \\
\hline \hline
\end{tabular}

\section{A. Classification Accuracy Analysis}

In order to test the reliability and efficiency of the proposed methodology, a validation dataset was compiled to produce oilspill and look-alike target database. As mentioned in Section III, the validation dataset consists of 110 feature vectors representing 100 oil spill and 10 look-alike spots identified by a visual interpretation technique. These 110 feature vectors contain different kinds of anomalies detected under a variety of sea conditions. The validation dataset is highly complex and in some cases, the image contains multiple look-alikes and oil spill spots. Here, it is important to note that most of the previously developed polarimetric oil spill detection algorithms were not tested extensively using a large dataset representing different situations and complexity. More often, image subscenes containing a single dark-spot representing an oil spill on a uniform background were taken into account, wherefore the limited complexity of such subscenes does not show the full capability of the algorithm (e.g., [15], [22]). The capabilities of the proposed algorithm have been tested on whole images rather than subsections of images. After processing all the images present in the validation dataset with the proposed algorithm, a detailed comparison with reference dataset was carried out on spot-by-spot basis by visual interpretation. The popular method of using a confusion matrix was employed for quantitative assessment of the proposed algorithm. Separate confusion matrices were constructed for each image, the framework of the confusion matrix is shown in Table II, similar to the evaluation technique used in [1]. 
TABLE III

FRAMEWORK OF THE CONFUSION MATRIX USED FOR QUANTITATIVE Assessment of THE PROPOSED AlgorithM Using ONLY TRADITIONAL Features (AlGO: Proposed Algorithm; REF: ReFERENCE DATASET)

\begin{tabular}{lcc}
\hline \hline & Oil Spill ALGO & Look-Alike ALGO \\
\hline Oil Spill REF & $A=82,(82 \%)$ & $B=18,(18 \%)$ \\
Look-Alike REF & $C=6,(60 \%)$ & $D=4,(40 \%)$ \\
\hline \hline
\end{tabular}

Explanation of the matrix in Table II (OS: Oil spill; LA: Look-alike)

A) OS(REF): OS(ALGO)—Number of co-located detections both by visual interpretation technique and proposed algorithm (ALGO).

B) OS(REF): LA(ALGO)—Dark spots classified as oil spills by visual interpretation technique but not by ALGO"False Negatives."

C) LA(REF): OS(ALGO)—Dark spots classified as oil spill by ALGO but not by visual interpretation technique "False Positives."

D) LA(REF): LA(ALGO)—Dark areas visually detected that could lead to false positives (look-alike). Classified as look-alike both by visual interpretation technique and ALGO, indication of degree of complexity present in particular image.

Although "B" and " $\mathrm{C}$ " both are the misclassified spots by proposed algorithm, " $\mathrm{B}$ " is considered to be the more serious misclassification event compared to "C." Table II shows overall classification accuracy and as well as individual accuracy assessment for oil spills and look-alikes, which indicates the degree of agreement between reference dataset and proposed algorithm. Although the test dataset is highly complex and contains multiple look-alikes and oil spills, agreement between the proposed algorithm and the reference dataset was found to be satisfactory. The object-based SVM classifier correctly identified $90 \%$ of oil spills (i.e., $A /(A+B)$ ) with overall classification accuracy of $89 \%$ (\% of correctly classified dark-spots, i.e., $(A+D) /(A+B+C+D))$ for a large validation dataset containing full-swath test images. Despite the satisfactory overall accuracy, one still has to consider the false positive rate of $20 \%$ in the case of combined features. However, the false positive rate for combined features is considerably lower compared to those of purely traditional and polarimetric features. As one of the main objectives of the study is to evaluate the performance of different kinds of feature parameters, we also carried out classification using only traditional (see Table III) and polarimetric (see Table IV) features separately. Using only traditional features, a classification accuracy of $82 \%$ and $40 \%$ was obtained for oil spill and look-alike, respectively, whereas using only polarimetric features a classification accuracy of $85 \%$ and $60 \%$ was obtained for oil spill and look-alike, respectively. After consulting Tables II, III, and IV, it is indisputable that a combination of traditional and polarimetric features improved the overall classification accuracy, especially in terms of detecting look-alike spots.
TABLE IV

FRAMEWORK OF THE CONFUSION MATRIX USED FOR QUANTITATIVE ASSESSMENT OF THE PROPOSED ALGORITHM USING ONLY POLARIMETRIC FEATURES (ALGO: Proposed Algorithm; REF: REFERENCE DATASET)

\begin{tabular}{lcc}
\hline \hline & Oil Spill ALGO & Look-Alike ALGO \\
\hline Oil Spill REF & $\begin{array}{c}A=85,(85 \%) \\
\text { Look-Alike REF }\end{array}$ & $\begin{array}{c}B=15,(15 \%) \\
D=4,(40 \%)\end{array}$ \\
\hline \hline
\end{tabular}

While it is challenging to compare different methodologies without using exactly the same dataset, a nonexhaustive comparison of our final results with previous work is carried out. It is important to note that only few recent studies reported some classification accuracy assessment and those methodologies are built on "pixel-based" classification techniques. Moreover, those studies exploited only polarimetric features and used a limited number of datasets and look-alike types, e.g., [6]. In [6], authors investigated the standard $k$-means classification technique using $\log \left(r_{\mathrm{CO}}\right)$ and $\log \left(\mu^{(d)}\right)$, where the Wishart classification of the covariance matrix was used as a reference dataset. Authors reported an overall classification accuracy of $79.8 \%$ using only $\log \left(r_{\mathrm{CO}}\right), 84.1 \%$ using only $\log \left(\mu^{(d)}\right)$, and $83.6 \%$ combining both $\log \left(r_{\mathrm{CO}}\right)$ and $\log \left(\mu^{(d)}\right)$.

\section{B. Discriminative Power of Polarimetric and Traditional Features}

As mentioned in the Section IV, we investigate the discriminative power of the features in terms of relevance and redundancy. The 25 features are listed in Table $\mathrm{V}$ in descending order of relevance (i.e., $\mathcal{I}(X \mid Y)$, mutual information of each feature $Y$ and the class property $X$ ). To remind the reader about the intuitive meaning of relevance, we restate that the ranking in Table $\mathrm{V}$ reflects the information content of the different features with respect to classification. A first general observation from Table $\mathrm{V}$ is that (dual-)polarimetric contrast features tend to have a stronger discriminative power than both classical (singlepol) intensity-based features and dual-polarimetric object feature values. Only the comparison of polarimetric features in objects against these values for background yields a high information content in terms of classification. From Table V, we also conclude that the only polarimetric contrast feature in the lower third is Rco_Contr. This finding corroborates the observation that the histogram of Rco_Contr in Fig. 5 has little discriminative power since it is strongly dominated by one point mass at Rco_Contr $=1.0$. However, this observation differs from the findings of [7] (a follow up study from [6]) where three dualpol TerraSAR-X scenes were evaluated. A possible explanation may lie in the fact that our study comprises a rather wide incidence angle range $\left(20^{\circ}\right.$ to $\left.35^{\circ}\right)$ along with various met-oceanic conditions as compared to the three TerraSAR-X scene used in [7] containing only one type of look-alike category (plant oil). On the other hand, our findings regarding Geom_Int_Contr $\left(\zeta_{\mu}\right)$ of being the most relevant feature closely matches the results obtained in [6] and [7]. Its important to note that this observation 
TABLE V

Relevance Values in Terms of Mutual Information $\mathcal{I}\left(Y_{i} \mid\right.$ CLASS $)$ For DifFERENT TRADITIONAL AND POLARIMETRIC FEATURES $Y_{i}$ AND ClasS Property Class, SORTED In DESCENDING ORder; No NORMALIZATION WAS CARRIED OUT

\begin{tabular}{|c|c|c|}
\hline Feature $Y_{i}$ (Derived From) & $\mathcal{I}\left(Y_{i} \mid\right.$ Class $)$ & Feature Category \\
\hline$Y_{19}=$ Geom_Int_Contr $\left(\mu^{(d)}\right)$ & 0.43435536 & Dual polarimetric-contrast \\
\hline$Y_{15}=$ Pw_Ratio_Contr $\left(\gamma_{\mathrm{CO}}\right)$ & 0.43030952 & Dual polarimetric-contrast \\
\hline$Y_{17}=$ Span_Contr $\left(\operatorname{Span}^{(d)}\right)$ & 0.41153182 & Dual polarimetric-contrast \\
\hline$Y_{14}=$ Pw_Ratio_Obj $\left(\gamma_{\mathrm{CO}}\right)$ & 0.39556791 & Dual polarimetric-object \\
\hline$Y_{23}=\mathrm{A} \_$Contr $\left(A^{(d)}\right)$ & 0.38074514 & Dual polarimetric-contrast \\
\hline$Y_{6}=$ Max_Contr $\left(\sigma_{0 \mathrm{Vv}}\right)$ & 0.37435768 & Traditional \\
\hline$Y_{5}=$ StdDev_Obj $\left(\sigma_{0 \mathrm{VV}}\right)$ & 0.37396138 & Traditional \\
\hline$Y_{24}=$ Alpha_Obj $\left(\alpha^{(d)}\right)$ & 0.37385888 & Dual polarimetric-object \\
\hline$Y_{9}=\mathrm{CPD} \_$Contr $\left(\sigma_{\varphi, \mathrm{CO}}\right)$ & 0.36981304 & Dual polarimetric-contrast \\
\hline$Y_{11}=$ Corr_Contr $\left(\rho_{\mathrm{CO}}\right)$ & 0.36836651 & Dual polarimetric-contrast \\
\hline$Y_{7}=$ Mean_Contr $\left(\sigma_{0} \mathrm{Vv}\right)$ & 0.36487212 & Traditional \\
\hline$Y_{3}=$ Complexity (Geometry) & 0.36091791 & Traditional \\
\hline$Y_{21}=\mathrm{H} \_$contr $\left(H^{(d)}\right)$ & 0.35548907 & Dual polarimetric-contrast \\
\hline$Y_{20}=\mathrm{H} \_$Obj $\left(H^{(d)}\right)$ & 0.34583747 & Dual polarimetric-object \\
\hline$Y_{10}=$ Corr_Obj $\left(\rho_{\mathrm{CO}}\right)$ & 0.34455698 & Dual polarimetric-object \\
\hline$Y_{25}=$ Alpha_Contr $\left(\alpha^{(d)}\right)$ & 0.33402118 & Dual polarimetric-contrast \\
\hline$Y_{8}=\mathrm{CPD} \_\mathrm{Obj}\left(\sigma_{\varphi, \mathrm{CO}}\right)$ & 0.31725307 & Dual polarimetric-object \\
\hline$Y_{22}=\mathrm{A} \_$Obj $\left(A^{(d)}\right)$ & 0.31501395 & Dual polarimetric-object \\
\hline$Y_{18}=$ Geom_Int_Obj $\left(\mu^{(d)}\right)$ & 0.28707119 & Dual polarimetric-object \\
\hline$Y_{13}=$ Rco_Contr $(R \mathrm{co})$ & 0.28407560 & Dual polarimetric-contrast \\
\hline$Y_{2}=$ Perimeter (Geometry) & 0.26252907 & Traditional \\
\hline$Y_{1}=$ Area (Geometry) & 0.24698244 & Traditional \\
\hline$Y_{16}=$ Span_Obj $\left(\operatorname{Span}^{(d)}\right)$ & 0.24439306 & Dual polarimetric-object \\
\hline$Y_{12}=$ Rco_Obj $(R \mathrm{co})$ & 0.21920133 & Dual polarimetric-object \\
\hline$Y_{4}=$ Spreading (Geometry) & 0.14384433 & Traditional \\
\hline
\end{tabular}

is only valid for X-band and the outcome form C-band might produce different result.

The object features Spreading, Rco_Obj, Span_Obj, and Geom_Int_Obj are likewise found to be of inferior relevance. A close look at the histograms in Figs. 9, 10, and 11 reveal that the overlap of occurrences of feature values for lookalike and oil spill throughout the feature range makes discrimination of classes by these features comparably difficult. Only the comparison with the background really gives the observer a greater insight into the possible class. We also conclude from Table V that most of the nonpolarimetric features are less relevant than the polarimetric contrast features, emphasizing the importance of using dual-pol data in such applications.

To interpret the redundancy (see the definition in Section IV), the readers are reminded that this value indicates how similar the information content of two features is, i.e., how much informational overlap the features have. This is not to be confused with the information content of the individual features. Concerning this redundancy, we computed the mutual information for all 25 mentioned features. In order to interpret the results in a comprehensive way, we displayed the normalized mutual information $\left(\mathcal{I}_{\text {norm }}(X ; Y)=\mathcal{I}(X ; Y) /(\sqrt{\mathcal{H}(X)} \sqrt{\mathcal{H}(Y)})\right.$ in a color coded matrix (see Fig. 8). $\mathcal{H}(Y)$ is defined as the statistical entropy of a random variable $Y$ by $\mathcal{H}(Y)=\sum_{y \in Y} p(y) \log (p(y))$. The features that appear to be highly uncorrelated (in terms of information theory) to all other features are Rco_Contr, Rco_Obj, Span_Obj, and Geom_Int_Obj. Keeping in mind that the distribution of Rco_Cont is highly concentrated around a certain value, the low correlation (in terms of information theory) comes

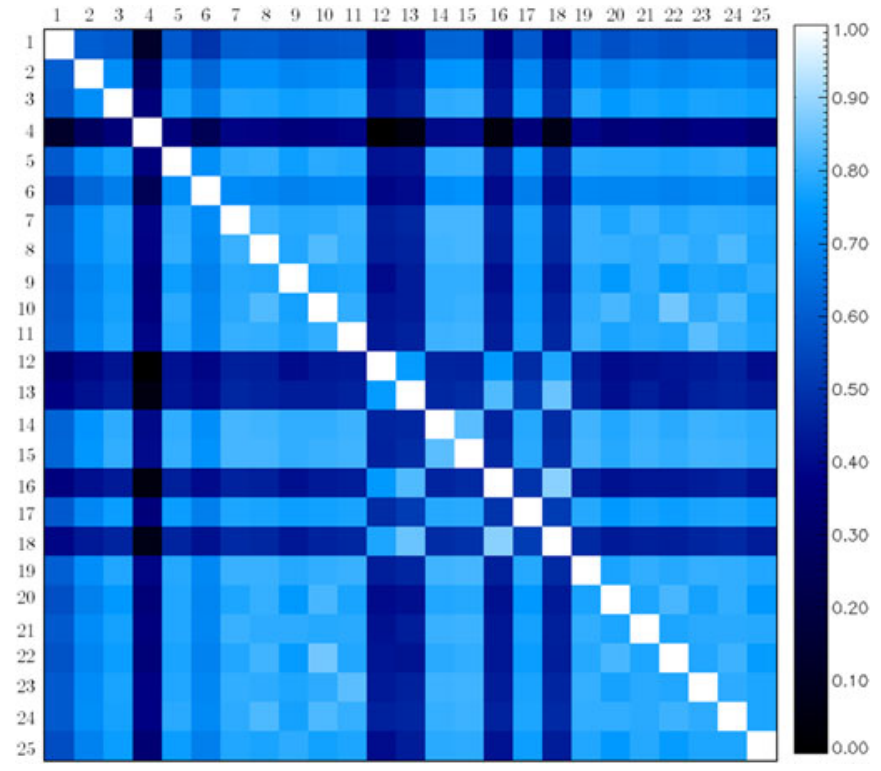

Fig. 8. Normalized mutual information $\mathcal{I}_{\text {norm }}(X \mid Y)=\mathcal{I}(X \mid Y) /$ $(\sqrt{\mathcal{H}(X)} \sqrt{\mathcal{H}(Y)}$ of polarimetric features $X, Y$. Color scale ranges from black (0.0) to white (1.0), where lighter tones of blue indicate a higher value of $\mathcal{I}_{\text {norm }}$. 1 Area, 2 Perimeter, 3 Complexity, 4 Spreading, 5 Std_Dev_Obj, 6 Max_Contr, 7 Mean_Contr, 8 CPD_Obj, 9 CPD_Contr, 10 Corr_Obj, 11 Corr_Contr, 12 Rco_Obj, 13 Rco_Contr, 14 Pw_Ratio_Obj, 15 Pw_Ratio_Contr, 16 Span_Obj, 17 Span_Contr, 18 Geom_Int_Obj, 19 Geom_Int_Contr, 20 H_Obj, 21 H_contr, 22 A_Obj, 23 A_Contr, 24 Alpha_Obj, 25 Alpha_Contr.

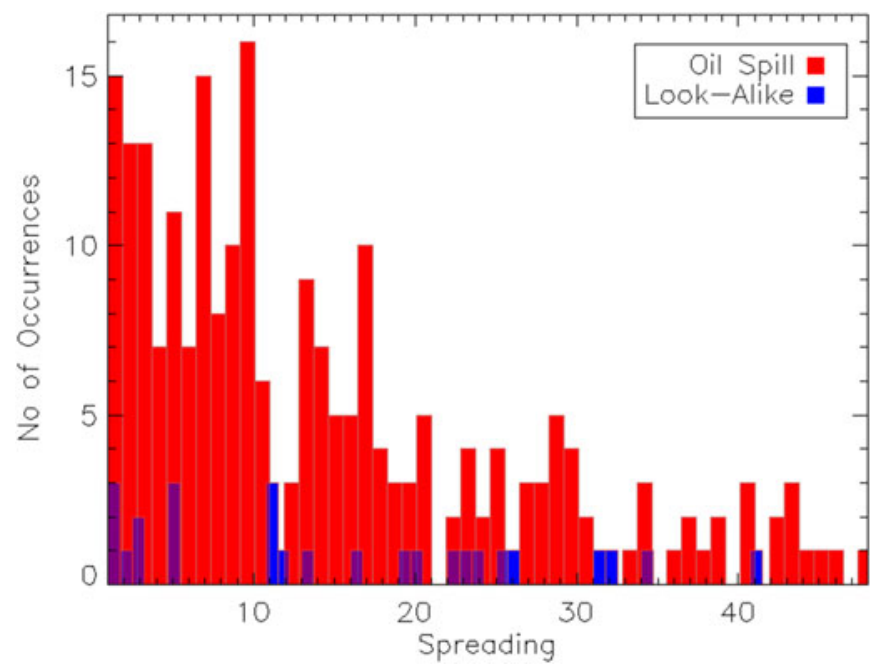

Fig. 9. Histogram of spreading (traditional feature).

at no surprise but owes to this very distributional characteristic. However, since we found the relevance of Rco_Contr to be relatively low, this does not imply that it carries valuable unique information. The histogram of Rco_Obj exhibits a similar characteristic that is dominated by contributions into one particular bin (see Fig. 5). So, an analogous argument concerning (information) correlation of Rco_Obj holds. Both Rco-based features are (in terms of information) unrelated to all other features which may very likely be due to mentioned dominating point mass (see above discussion for low relevance of Rco-based features). The 


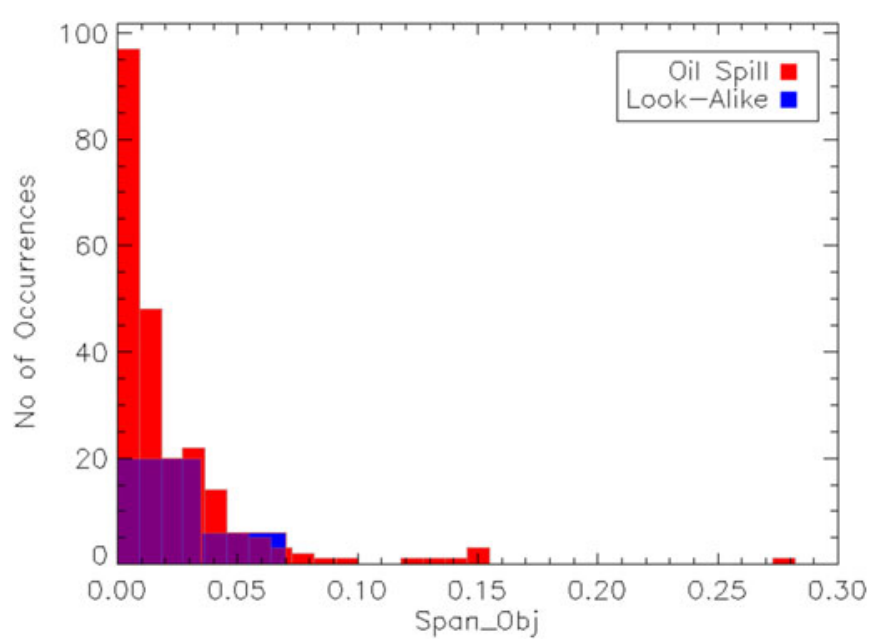

Fig. 10. Histogram of Span_Obj.

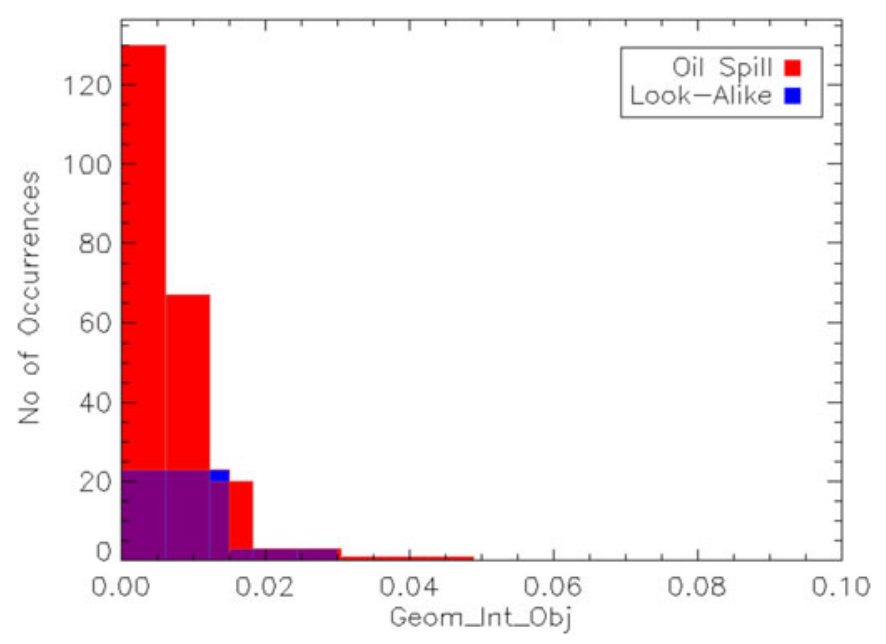

Fig. 11. Histogram of Geom_Int_Obj feature.

low correlation of Spreading, Span_Obj, and Geom_Int_Obj does not have an explanation as direct as that for the Rcobased features. What can also be directly inferred from Fig. 8 is that most polarimetric features (except for Span_Obj and Geom_Int_Obj) share a lot of common information, i.e., their may well be a high degree of redundancy among them (entries of the lower right part of the matrix are greater than 0.70). From Fig. 8, we cannot, however, conclude that any particular features can be discarded because of high redundancy. Empirical tests, where one classifies with a reduced subset of features to reduce computational overhead, may still prove that certain features are discardable. This has to be investigated in future work.

\section{CONCLUSION}

A combination of traditional and polarimetric features is exploited for the first time to characterize dark spots on Xband coherent dual-polarization SAR data using support vector machine. Classification accuracy assessment shows our proposed methodology correctly identifies $90 \%$ oil spills and $80 \%$ look-alikes from validation dataset with an overall accuracy of $89 \%$. Combining traditional and polarimetric features improved the classification accuracy compared to classification results obtained from traditional and polarimetric features separately. The contributions of geometric intensity contrast $\left(\zeta_{\mu}\right)$, Copolarization Power ratio contrast $\left(\zeta_{\gamma_{\mathrm{CO}}}\right)$, span contrast $\left(\zeta_{\mathrm{span}}\right)$ and eigenvalue-based polarmetric features to the classification stage are most noteworthy, along with some traditional features like backscatter contrast between object and background. These features clearly deserve attention in future studies, in particular whether they produce a statistically significant improvement of classification on a much larger sample array. Additionally, the proposed methodology is tuned for operational NRT services with average processing time of 7 $-8 \mathrm{~min}$ for a standard dual pol TS-X StripMAP scene. Initial evaluation of the classifier shows considerable improvements over the classifiers based only on traditional features. It has been shown that a combination of traditional and polarimetric features needs to be utilized in order to develop a robust classifier. In particular, contrast-based polarimetric features exhibit outstanding classification relevance that can add to the classification quality. A first analysis of redundancy among these features indicates the directions for further investigation on selection of optimal features combinations (which can help to avoid computational and memory overhead). An extensive evaluation of features and performance estimation of the classifier for $\mathrm{C}$ and $\mathrm{L}$ band polarimetric SAR images in different environmental conditions is foreseen in the near future.

\section{ACKNOWLEDGMENT}

The authors would like to thank anonymous reviewers for their constructive comments. TerraSAR-X images were acquired through Science AO OCE 2015 (PI: S. Singha) and OCE 1045 (PI: D. Velotto). The views, opinions, and findings contained in this paper are those of the authors and should not be construed as an official DLR position, policy or decision.

\section{REFERENCES}

[1] S. Singha, M. Vespe, and O. Trieschmann, "Automatic synthetic aperture radar based oil spill detection and performance estimation via a semiautomatic operational service benchmark," Marine Pollut. Bull., vol. 73, no. 1, pp. 199-209, Aug. 2013.

[2] K. N. Topouzelis, "Oil spill detection by SAR images: Dark formation detection, feature extraction and classification algorithms," Sensors, vol. 8, no. 10, pp. 6642-6659, 2008.

[3] M. Migliaccio, A. Gambardella, and M. Tranfaglia, "SAR polarimetry to observe oil spills," IEEE Trans. Geosci. Remote Sens., vol. 45, no. 2, pp. 506-511, Feb. 2007.

[4] M. Migliaccio, F. Nunziata, A. Montuori, X. Li, and W. G. Pichel, "A multifrequency polarimetric SAR processing chain to observe oil fields in the Gulf of Mexico," IEEE Trans. Geosci. Remote Sens., vol. 49, no. 12, pp. 4729-4737, Dec. 2011.

[5] M. Migliaccio, F. Nunziata, and A. Gambardella, "On the co-polarized phase difference for oil spill observation," Int. J. Remote Sens., vol. 30, pp. 1587-1602, 2009.

[6] S. Skrunes, C. Brekke, and T. Eltoft, "Characterization of marine surface slicks by Radarsat-2 multipolarization features," IEEE Trans. Geosci. Remote Sens., vol. 52, no. 9, pp. 5302-5319, Sep. 2014.

[7] S. Skrunes, C. Brekke, T. Eltoft, and V. Kudryavtsev, "Comparing nearcoincident $\mathrm{C}$-and X-band SAR acquisitions of marine oil spills," IEEE Trans. Geosci. Remote Sens., vol. 53, no. 4, pp. 1958-1975, Apr. 2015. 
[8] V. N. Kudryavtsev, B. Chapron, A. G. Myasoedov, F. Collard, and J. A. Johannessen, "On dual co-polarized SAR measurements of the ocean surface," IEEE Geosci. Remote Sens. Lett., vol. 10, no. 4, pp. 761-765, Jul. 2013.

[9] D. Velotto, F. Nunziata, M. Migliaccio, and S. Lehner, "Dual-polarized TerraSAR-X data for oil-spill observation," IEEE Trans. Geosci. Remote Sens., vol. 49, no. 12, pp. 1114-1118, Dec. 2011.

[10] F. Nunziata, M. Migliaccio, and A. Gambardella, "Pedestal height for sea oil slick observation," IET Radar Sonar Navig., vol. 5, no. 2, pp. 103-110, Feb. 2011.

[11] B. Minchew, C. Jones, and B. Holt, "Polarimetric analysis of backscatter from the deepwater horizon oil spill using 1-band synthetic aperture radar," IEEE Trans. Geosci. Remote Sens., vol. 50, no. 10, pp. 3812-3830, Oct. 2012.

[12] B. Zhang, W. Perrie, X. Li, and W. G. Pichel, "Mapping sea surface oil slicks using radarsat-2 quad-polarization SAR image," Geophys. Res. Lett., vol. 38, no. 10, 2011, Art. no. 110602. [Online]. Available: http://dx.doi.org/10.1029/2011GL047013

[13] A. H. S. Solberg, "Remote sensing of ocean oil-spill pollution," Proc. IEEE, vol. 100, no. 10, pp. 2931-2945, Oct. 2012.

[14] M. Gade, W. Alpers, H. Hühnerfuss, H. Masuko, and T. Kobayashi, "Imaging of biogenic and anthropogenic ocean surface films by the multifrequency/multipolarization SIR-C/X-SAR," J. Geophys. Res., vol. 103, no. C9, pp. 18851-18866, 1998.

[15] F. Nunziata, A. Gambardella, and M. Migliaccio, "On the Mueller scattering matrix for SAR sea oil slick observation," IEEE Geosci. Remote Sens. Lett., vol. 5, no. 4, pp. 691-695, Oct. 2008.

[16] S. R. Cloude and E. Pottier, "A review of target decomposition theorems in radar polarimetry," IEEE Trans. Geosci. Remote Sens., vol. 34, no. 2, pp. 498-518, Mar. 1996.

[17] S. R. Cloude and E. Pottier, "An entropy based classification scheme for land applications of polarimetric SAR," IEEE Trans. Geosci. Remote Sens., vol. 35, no. 1, pp. 68-78, Jan. 1997.

[18] I. Hajnsek, E. Pottier, and S. R. Cloude, "Inversion of surface parameters from polarimetric SAR," IEEE Trans. Geosci. Remote Sens., vol. 41, no. 4, pp. 727-744, Apr. 2003.

[19] S. R. Cloude and E. Pottier, "An entropy based classification scheme for land applications of polarimetric SAR," IEEE Trans. Geosci. Remote Sens., vol. 35, no. 1, pp. 68-78, Jan. 1997.

[20] J. Praks, E. Koeniguer, and M. Hallikainen, "Alternatives to target entropy and alpha angle in SAR polarimetry," IEEE Trans. Geosci. Remote Sens., vol. 47, no. 7, pp. 2262-2274, Jul. 2009.

[21] M. Migliaccio, A. Gambardella, F. Nunziata, M. Shimada, and O. Isoguchi, "The PALSAR polarimetric mode for sea oil slick observation," IEEE Trans. Geosci. Remote Sens., vol. 47, no. 12, pp. 4032-4041, Dec. 2009.

[22] P. Liu, X. Li, J. J. Qu, W. Wang, C. Zhao, and W. Pichel, "Oil spill detection with fully polarimetric UAVSAR data," Marine Pollution Bull., vol. 62, no. 12, pp. 2611-2618, 2011.

[23] S. Singha, D. Velotto, and S. Lehner, "Near real time monitoring of platform sourced pollution using TerraSAR-X over the North Sea," Marine Pollution Bull., vol. 86, no. 1, pp. 379-390, 2014.

[24] X. ming Li and S. Lehner, "Algorithm for sea surface wind retrieval from TerraSAR-X and TanDEM-X data," IEEE Trans. Geosci. Remote Sens., vol. 52, no. 5, pp. 2928-2939, May 2014.

[25] A. Gambardella, G. Giacinto, M. Migliaccio, and A. Montali, "One-class classification for oil spill detection," Pattern Anal. Appl., vol. 13, no. 3, pp. 349-366, 2010. [Online]. Available: http://dx.doi.org/10.1007/s10044009-0164-Z

[26] J. M. Moguerza and A. Muñoz, "Support vector machines with applications," Stat. Sci., pp. 322-336, vol. 21, no. 3, 2006.

[27] L. Xu, J. Li, and A. Brenning, "A comparative study of different classification techniques for marine oil spill identification using RADARSAT-1 imagery," Remote Sens. Environ., vol. 141, pp. 14-23, 2014.

[28] L. Yu and H. Liu, "Feature selection for high-dimensional Data: A fast correlation-based filter solution," in Proc. 20th Int. Conf. Mach. Learn., 2003, pp. 856-863.

[29] H. Peng, F. Long, and C. Ding, "Feature selection based on mutual information criteria of max-dependency, max-relevance, and min-redundancy," IEEE Trans. Pattern Anal. Mach. Intell., vol. 27, no. 8, pp. 1226-1238, Aug. 2005.

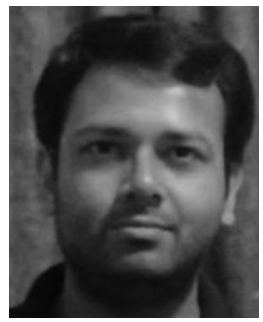

Suman Singha (M'15) received the M.Tech. and M.Sc. degrees in remote sensing from the Indian Institute of Technology, Roorkee, India and the University of Hull, United Kingdom, in 2009 and 2010, respectively, and the $\mathrm{Ph} . \mathrm{D}$. degree in microwave remote sensing from the University of Hull, Hull, U.K., in 2014.

From March 2012 to July 2012, he was a Visiting Scientist with the European Maritime Safety Agency, Portugal. Since 2013, he has been with the Maritime Safety and Security Lab of the German Aerospace Center, Bremen, Germany, as a Research Scientist. His research interests include the application of artificial intelligence and machine vision approaches to classify traditional and polarimetric SAR images with emphasis on oil spill detection and sea ice classification.

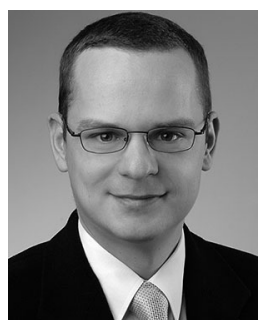

Rudolf Ressel (M'15) received the Diploma degree in mathematics from the Georg-August-University, Göttingen, Germany, and the Ph.D. degree in mathematics from the University of Bremen, Bremen, Germany, in 2008 and 2012, respectively, in the field of inverse problems.

In July 2013, he joined the German Aerospace Center (DLR), where he works as Research Scientist with the Maritime Safety and Security Laboratory, Bremen, Germany. His main research interests include ice type classification by means of SAR image

analysis.

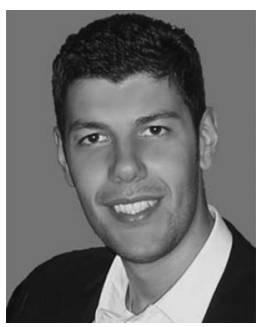

Domenico Velotto (M'15) was born in Italy, on April 30, 1981. He received the M.S. degree (5-years legal course of study) in nautical science (curriculum electronic radio-navigation) from the Università degli Studi di Napoli, "Parthenope", Napoli, Italy, in 2008. In 2009, he started working toward the Ph.D degree at the Synthetic Aperture Radar Oceanography Group, Remote Sensing Technology Institute (IMF), German Aerospace Center, Weßling, Germany.

Since 2013, he has been working as a Research Assistant at the Maritime Safety and Security Lab, DLR, Bremen, Germany. His main research interests include electromagnetic models, image processing and Earth observation with SAR polarimetry with emphasis on oil spill and maritime targets detection and classification.

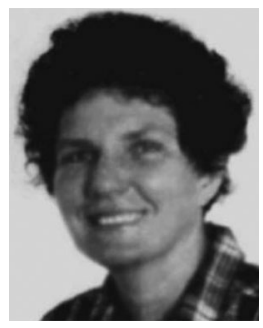

Susanne Lehner (M'01) received the M.Sc. degree in applied mathematics from Brunel University, Uxbridge, U.K., and the Ph.D. degree in geophysics from the University of Hamburg, Hamburg, Germany, in 1979 and 1984, respectively.

She was a Research Scientist with the Max-Planck Institute for Climatology, Hamburg, Germany, and joined the German Aerospace Center (DLR/DFD), Wessling, Germany, in 1996. She is currently with the Maritime Safety and Security Lab, Remote Sensing Technology Institute (IMF), German Aerospace Center (DLR), working on the development of algorithms determining marine parameters from synthetic aperture radars. 